\title{
Addition of Sodium Pyruvate to Stored Red Blood Cells Attenuates Liver Injury in a Murine Transfusion Model
}

\author{
Sha Xia, Gan Chen, Bo Wang, Yujing Yin, Zhenwei Sun, Jingxiang Zhao, \\ Penglong Li, Lian Zhao, and Hong Zhou
}

Beijing Institute of Transfusion Medicine, Beijing Key Laboratory of Blood Safety and Supply Technologies, No. 27 Taiping Road, Haidian, Beijing, China

Correspondence should be addressed to Lian Zhao; zhaolian@bmi.ac.cn and Hong Zhou; zhouhtt1966@163.com

Received 11 April 2016; Revised 20 July 2016; Accepted 1 September 2016

Academic Editor: Vera L. Petricevich

Copyright $(9) 2016$ Sha Xia et al. This is an open access article distributed under the Creative Commons Attribution License, which permits unrestricted use, distribution, and reproduction in any medium, provided the original work is properly cited.

\begin{abstract}
RBCs undergo numerous changes during storage and stored RBCs may induce adverse effects, ultimately resulting in organ injury in transfusion recipients. We tested the hypothesis that the addition of SP to stored RBCs would improve the quality of the stored RBCs and mitigate liver injury after transfusion in a murine model. RBCs were harvested from C57BL/6J mice and stored for 14 days in CPDA-1 containing either a solution of SP in saline or saline alone. Haemolysis, the 24-hour posttransfusion recovery, the oxygen-carrying capacity, and the SOD activity of stored RBCs were evaluated. The plasma biochemistry, hepatic MDA level, MPO activity, IL-6, TNF- $\alpha$ concentrations, and histopathology were measured two hours after the transfusion of stored RBCs. Compared with RBCs stored in CPDA-1 and saline, the addition of SP to stored RBCs restored their oxygen-carrying capacity and SOD activity, reduced the AST activity, BUN concentrations, and $\mathrm{LDH}$ activity in the plasma, and decreased the MDA level, MPO activity, and concentrations of IL- 6 and TNF- $\alpha$ in the liver. These data indicate that the addition of SP to RBCs during storage has a beneficial effect on storage lesions in vitro and subsequently alleviates liver injury after the transfusion of stored RBCs in vivo.
\end{abstract}

\section{Introduction}

Red blood cell (RBC) transfusion is a lifesaving therapy for patients with haemorrhagic shock, anaemia, and surgery. To augment utilization and minimize waste, RBCs are often refrigerated and stored for several days in clinical situations. During storage, RBCs undergo a series of structural and functional alterations, referred to as the "storage lesion" [13]. Cellular ATP and 2,3-DPG depletion have been observed during storage, and these changes influence the survival of transfused RBCs and their oxygen-carrying capacity [46]. Additionally, the loss of cellular antioxidation capacity results in the generation of reactive oxygen species (ROS) during storage, which increases protein and lipid oxidation in stored RBCs [7]. This damage may directly stimulate the production of inflammation mediators and subsequently cause organ injury including liver injury in some recipient after the transfusion of stored RBCs $[8,9]$, which may be associated with increased morbidity and mortality [10-12].
The Food and Drug Administration (FDA) has approved the storage of RBCs in storage solutions. Specifically, RBCs can be stored for up to 42 days without exceeding $1 \%$ haemolysis and a minimum of $75 \%$ of transfused RBCs survival $24 \mathrm{~h}$ after transfusion [13-15]. Many studies have attempted to reduce storage lesions by improving the composition of the storage solution. The addition of nutrients and antioxidant was previously demonstrated to alleviate storage lesions during RBC storage. The addition of exogenous glucose can extend the storage of RBCs by decreasing haemolysis and supplying energy $[16,17]$. Stowell et al. indicated that the addition of ascorbic acid solution benefited the recovery and immunogenicity of RBCs during storage [4]. This study also indicated that simple antioxidants could not decrease inflammation in a murine transfusion model. Herein, we hypothesized that a multifunctional additive solution can provide a substrate for energy metabolism and exert an antioxidant effect during RBC storage which can reduce storage lesions as well as decrease oxidative stress and inflammation after transfusion in a murine transfusion model. 
Pyruvate is a three-carbon molecule that is endogenously produced during glycolysis. Pyruvate can be converted into ATP in the tricarboxylic acid (TCA) cycle and has also been demonstrated to act as an antioxidant [18]. Sodium pyruvate (SP) was reported to attenuate tissue damage in haemorrhagic shock, brain damage, and ischemia-reperfusion injury models [19-22]. In addition, previous studies indicated that pyruvate can maintain ATP and 2,3-DPG levels during RBC storage in vitro $[23,24]$, and these results suggested that the addition of SP during RBC storage might alleviate storage lesions, which need to be further studied. Moreover, the effects of adding SP during RBC storage on organ injury after the transfusion of stored RBCs in vivo are unknown. We hypothesized that the addition of SP during RBC storage can reduce storage lesions and subsequently decrease organ injury in recipients after transfusion.

The murine storage RBC model is widely used to study the damage to RBCs during storage $[9,25]$. Previous studies showed that murine RBCs stored for two weeks were similar to human RBCs stored for six weeks and underwent similar physiological and biochemical changes during storage $[7,26-$ 28]. The effects of SP on the transfusion of stored RBCs were evaluated in a murine model. We found that SP can restore the oxygen-carrying capacity of RBCs and decrease oxidative stress and inflammation in the liver after the transfusion of stored RBCs in a murine model.

\section{Materials and Methods}

2.1. Animals. The study was approved by the Institutional Animal Care and Use Committee of the Academy of Military Medical Sciences (IACUC number AMMS-2014-027). All efforts were made to minimize the number of animals used and their suffering. Eight- to ten-week-old wild-type male C57BL/6J mice were purchased from Vital River (Beijing, China) and used after an acclimation period of at least five to seven days at $25^{\circ} \mathrm{C}$ in a $12 \mathrm{~h}$ light $/ 12 \mathrm{~h}$ dark cycle.

2.2. SP Stock Preparation and Addition. SP was purchased (Sigma, St. Louis, MO, USA), and stock solutions of SP were prepared in saline solution at final concentrations of $325 \mathrm{mmol} / \mathrm{L}$ and filtered through a $0.22 \mu \mathrm{m}$ vacuum filter. The SP stock solutions were then added to mouse RBCs at a $1: 130(\mathrm{vol} / \mathrm{vol})$ ratio, and the final concentration of SP in the storage solutions was $2.5 \mathrm{mmol} / \mathrm{L}$ prior to centrifugation.

2.3. Mouse RBCs: Collection, Storage, and Transfusion. The mice were anesthetized with intraperitoneal injections of sodium pentobarbital $(75 \mathrm{mg} / \mathrm{kg})$ and mechanically ventilated at a respiratory rate of $120 / \mathrm{min}$ and tidal volume of $10 \mu \mathrm{L} / \mathrm{g}$ using a Minivent mouse ventilator (Harvard Apparatus, Hugstetten, Germany). The body temperature was maintained at $36.5 \pm 0.5^{\circ} \mathrm{C}$ using a heating pad (SOFTRON, TMS-201, Beijing, China). During the blood collection, surgical site was disinfected with $75 \%$ ethanol, and the mice were aseptically bled by cardiac puncture at sterile work tables, and the blood was collected in CPDA-1 (Sigma, St. Louis, MO, USA). The final CPDA-1 concentration used for storage was $14 \%$. The whole blood collected from 20 to 30 mice was pooled, leukoreduced using a sterile high-efficiency leukoreduction filter (ZhiXing Bio S\&T Co., Bengbu, China), centrifuged at $400 \times \mathrm{g}$ for $15 \mathrm{~min}$, and volume-reduced to a final hematocrit of $70 \%$ to $75 \%$ ( $\mathrm{Hb}$ of 17 to $18 \mathrm{~g} / \mathrm{dL}$ ).

The RBCs were stored in two different solutions: (1) control group, RBCs stored in CPDA-1 plus saline, and (2) SP group, RBCs stored in CPDA-1 plus $2.5 \mathrm{mM}$ SP. The RBCs were placed in $0.6 \mathrm{~mL}$ tubes (Corning, CA, USA) and stored at $4^{\circ} \mathrm{C}$ for up to 14 days.

Before transfusion, the stored RBCs were washed three times using 10 volumes of PBS and centrifuged at $400 \times \mathrm{g}$. After the final wash, the washed and stored RBCs were resuspended in PBS to a final $\mathrm{Hb}$ concentration similar to a final hematocrit of $70 \%$ to $75 \%$. All recipient mice received $20 \%$ by volume of the total blood volume of the mouse via the tail vein. Two hours later, the mice were aseptically bled by cardiac puncture to assess the plasma biochemistry. The mice were then euthanized to obtain tissue samples. The tissue samples were washed with cold saline, snap-frozen in liquid nitrogen, and stored in liquid nitrogen until assayed.

2.4. Measurement and Calculations of In Vitro RBC Haemolysis. The blood gas was measured with an ABL90 FLEX Blood Gas Analyser (Radiometer, Copenhagen, Denmark) after 14 days of storage. The supernatant and total $\mathrm{Hb}$, which were used to calculate the percentage of haemolysis, were measured using a free haemoglobin assay kit (Jiancheng Bioengineering Institute, Nanjing, China). The haemolysis rate was subsequently calculated as follows [29]:

$$
\begin{aligned}
& \text { Haemolysis rate }(\%) \\
& \qquad=\frac{[(1-\mathrm{Hct}) \times \text { supernatant } \mathrm{Hb}(\mathrm{g} / \mathrm{dL})]}{\text { Total } \mathrm{Hb}(\mathrm{g} / \mathrm{dL})} \times 100 \% .
\end{aligned}
$$

2.5. Flow Cytometry Assessment of RBC Survival. RBCs were labelled with fluorescein isothiocyanate (FITC) (Sigma, St. Louis, MO, USA) as previously reported [30, 31]. A fluorescent dye solution was prepared by dissolving $100 \mathrm{mg}$ of fluorescein isothiocyanate (FITC) in $10 \mathrm{~mL}$ of PBS. This solution was added to the diluent stored blood, for a final FITC concentration of $66 \mu \mathrm{g} / \mathrm{mL}$. Cells were incubated in this staining solution for $20 \mathrm{~min}$ at $37^{\circ} \mathrm{C}$ in the dark, and the labelled RBCs were washed three times in PBS and transfused into each recipient mouse (five mice per experimental group) via the tail vein. Ten minutes and $24 \mathrm{~h}$ after the transfusion, the recipients were retroorbitally bled into heparinized tubes, and $5 \mu \mathrm{L}$ of blood was diluted in $1 \mathrm{~mL}$ of FACS buffer. The percentage of fluorescent RBCs was measured by flow cytometry (BD FACSCalibur, MA, USA) using the percentage of fluorescent RBCs measured 10 min after transfusion as the reference value. The $24 \mathrm{~h}$ survival was determined based on the ratio of fluorescent RBCs at $24 \mathrm{~h}$ to the reference value.

2.6. Haemoglobin Oxygen Saturation Experiments. Blood oxygen saturation was measured with a Hemox Analyser (TCS Scientific Corporation, New Hope, PA, USA). Briefly, a suspension of erythrocytes containing $6 \mathrm{mg}$ of haemoglobin was initially incubated at $37^{\circ} \mathrm{C}$ in $4 \mathrm{~mL}$ of 
Hemox buffer (30 mM TES (N-Tris hydroxymethyl-methyl2-aminoethanesulfonic acid), $135 \mathrm{mM} \mathrm{NaCl}$ and $5 \mathrm{mM} \mathrm{KCl}$, pH $7.40 \pm 0.02$ at $37^{\circ} \mathrm{C}$; osmolarity $295 \pm 10 \mathrm{mOsm} / \mathrm{kg}$ ) and $0.1 \%$ BSA in the Hemox Analyser. After the addition of $10 \mu \mathrm{L}$ of antifoaming agent (TCS Scientific, New Hope, PA, USA), the $\mathrm{pO}_{2}$ and temperature were allowed to stabilize before measuring the oxygen saturation. The $P_{50}$ value was then extrapolated on the $x$-axis as the point at which the $\mathrm{O}_{2}$ saturation was $50 \%$.

2.7. Measurements of Plasma Biochemistry and Lactate Levels. The plasma aspartate aminotransferase (AST) activity, blood urea nitrogen (BUN) concentration, and lactate dehydrogenase (LDH) activity were evaluated using a Hitachi 7180 autoanalyser (Hitachi High-Technologies Corp., Tokyo, Japan). The lactate concentration was determined by lactic acid assay kit (Jiancheng Biological Institute, Nanjing, China) according to the manufacturer's recommendations.

2.8. Measurement of MDA Level, MPO Activity, and SOD Activity. The activity of SOD in stored RBCs was measured using a SOD Assay Kit (Jiancheng Biological Institute, Nanjing, China). Liver tissue was homogenized in cold normal saline to prepare a $10 \%$ homogenate and then centrifuged at $1,000 \mathrm{rpm}$ and at $4^{\circ} \mathrm{C}$ for $6 \mathrm{~min}$. The supernatant was then collected to measure the MDA levels and MPO activity using MDA concentration and MPO activity assay kits (Jiancheng Biological Institute, Nanjing, China) according to the manufacturer's recommendations, as previously reported [32]. The total hepatic protein levels were measured using a BCA protein assay kit (Biomed Biology Corporation, Beijing, China).

2.9. Measurements of Inflammatory Cytokines Levels. Liver tissue was homogenized on ice in $0.9 \%$ saline containing a protease inhibitor cocktail (Roche, Mannheim, Germany). The homogenates were centrifuged at $1000 \times \mathrm{g}$ for $6 \mathrm{~min}$ at $4^{\circ} \mathrm{C}$, and the supernatants were assayed for inflammatory cytokines levels. The interleukin- (IL-) 6 and TNF- $\alpha$ levels in supernatant were determined using an enzyme-linked immunosorbent assay (ELISA) kit (PeproTech, Rocky Hill, NJ, USA) according to the manufacturer's instructions as reported [25]. The values are expressed as pg/mg protein.

2.10. Liver Histology. Two hours after transfusion, the livers were fixed with $4 \%$ paraformaldehyde and embedded in paraffin. The sections were stained with hematoxylin and eosin $(\mathrm{H} \& \mathrm{E})$ to observe histological changes [33]. The histological changes in the liver were examined by light microscopy in a blinded fashion. The liver injury was assessed in the inflammatory cell infiltration and scored on a 4-point scale ( 0 , none; 1 , slight; 2 , moderate; 3 , severe) as previously described [34].

2.11. Statistical Analysis. The data are presented as the means \pm standard deviations (SD). All analyses were conducted using statistical software (SAS Institute Inc., Cary, NC, USA). The sample numbers were chosen based on the mean values of the parameters from preliminary experiment.
TABLE 1: Blood gas of the RBCs after 14 days of storage $(n=4)$.

\begin{tabular}{lcc}
\hline Index & Control group & SP group \\
\hline $\mathrm{pO}_{2}(\mathrm{mmHg})$ & $77.5 \pm 3.9$ & $78.4 \pm 2.4$ \\
$\mathrm{pCO}_{2}(\mathrm{mmHg})$ & $134 \pm 5.3$ & $140 \pm 5.1$ \\
$\mathrm{sO}_{2}(\%)$ & $48.6 \pm 2.0$ & $44.7 \pm 1.8$ \\
$\mathrm{CHb}(\mathrm{g} / \mathrm{dL})$ & $24.12 \pm 1.3$ & $23.8 \pm 1.2$ \\
$\mathrm{Hct}(\%)$ & $73.8 \pm 3.9$ & $72.7 \pm 2.6$ \\
$\mathrm{Na}^{+}(\mathrm{mmol} / \mathrm{L})$ & $91.5 \pm 2.0$ & $92.1 \pm 1.6$ \\
$\mathrm{Cl}^{-}(\mathrm{mmol} / \mathrm{L})$ & $78.3 \pm 2.8$ & $79.3 \pm 1.4$ \\
Lactate $(\mathrm{mmol} / \mathrm{L})$ & $25.1 \pm 0.7$ & $27.0 \pm 0.6^{*}$ \\
\hline
\end{tabular}

${ }^{*} p<0.05$ versus the control group.

Four biological replicates in in vitro study or ten mice per group in in vivo study were required with a type I error of 0.05 and at least a power of $80 \%$. The means of each group were compared using one-way analysis of variance (ANOVA) followed by the Student-Newman-Keuls test when the normality and homogeneity of variance assumptions were satisfied; otherwise, ANOVA followed by Student-NewmanKeuls multiple range test was applied. The significance level was set to $p<0.05$.

\section{Results}

3.1. Blood Gas Analysis. The $\mathrm{pH}, \mathrm{pCO}_{2}, \mathrm{pO}_{2}, \mathrm{SBE}, \mathrm{Na}^{+}$, and $\mathrm{Cl}^{-}$values did not significantly differ between the control and SP groups (Table 1). The lactate content was significantly higher in the SP group $(27.0 \pm 0.6 \mathrm{mmol} / \mathrm{L})$ than in the control group $(25.1 \pm 0.7)(p<0.05)$.

3.2. Haemoglobin Oxygen Saturation Experiments. $P_{50}$ value is the $\mathrm{pO}_{2}$ at which haemoglobin is half-saturated with oxygen. As shown in Figure 1, the $P_{50}$ value in the SP group $(23.9 \pm 1.5 \mathrm{mmHg})$ was significantly higher than that in the control group $(21.0 \pm 0.7 \mathrm{mmHg})(p<0.05)$.

3.3. SOD Activity. SOD is an antioxidant enzyme that has been suggested to be important for ROS elimination. The SOD activity in stored RBCs was significantly higher in the SP group (726.8 $\pm 12.3 \mathrm{U} / \mathrm{mg}$ protein) than in the control group after storage for 14 days $(648.1 \pm 10.0 \mathrm{U} / \mathrm{mg}$ protein $)(p<0.05$, Figure 2).

3.4. Haemolysis and RBC $24 \mathrm{~h}$ Recovery. The haemolysis and $24 \mathrm{~h}$ recovery of the stored $\mathrm{RBCs}$ were measured. The haemolysis of stored RBCs in the SP group $(0.77 \pm 0.04 \%)$ was lower than that in the control group $(0.79 \pm 0.03 \%)$, but this difference was not significant $(p>0.05)$.

The $24 \mathrm{~h}$ recovery of stored RBCs was higher in the SP group $(77.86 \pm 5.4 \%)$ than in the control group $(75.63 \pm 1.8 \%)$, but this difference was also not significant $(p>0.05)$.

3.5. Plasma Biochemistry. The plasma biochemistry was investigated in this study. As shown in Figure 3(a), the activity of AST was significantly lower in the SP group (332 \pm 111.2 U/L) than in the control group $(579 \pm 96.5 \mathrm{U} / \mathrm{L})(p<$ 


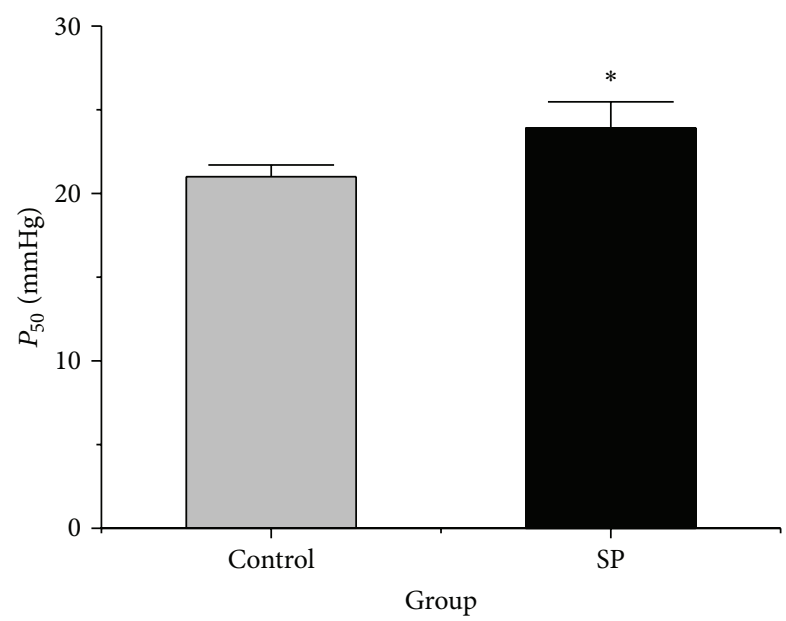

Figure 1: $P_{50}$ values in two groups $(n=4)$. The data are plotted as the means $\pm \mathrm{SD} .{ }^{*} p<0.05$ versus the control group.

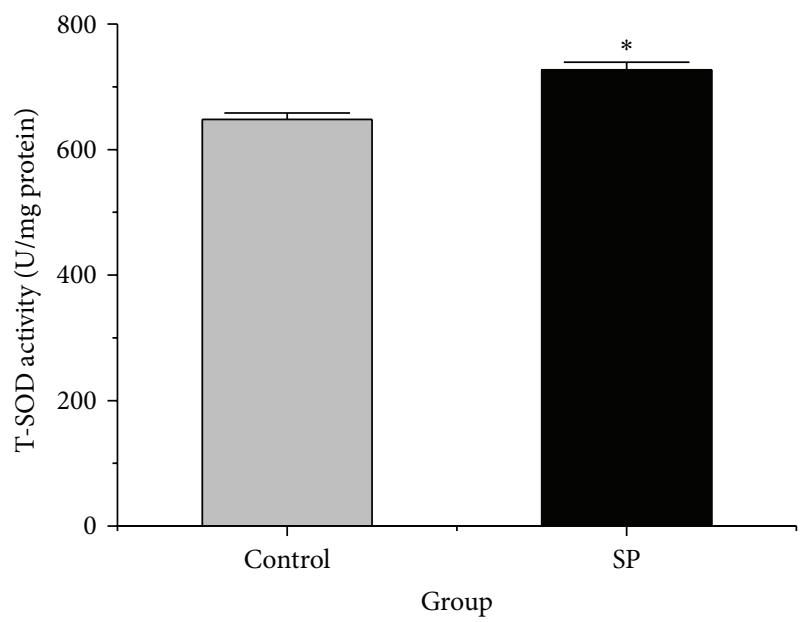

FIGURE 2: SOD activities of the two groups $(n=4)$. The data are plotted as the means \pm SD. ${ }^{*} p<0.05$ versus the control group.

0.05). The BUN concentration was also significantly lower in the SP group $(11.9 \pm 1.0 \mathrm{mmol} / \mathrm{L})$ than in the control group $(14.6 \pm 0.8 \mathrm{mmol} / \mathrm{L})(p<0.05$, Figure $3(\mathrm{~b}))$. The LDH activity was also significantly lower in the SP group $(368.8 \pm 72.5 \mathrm{U} / \mathrm{L})$ than in the control group $(479.0 \pm 101.6 \mathrm{U} / \mathrm{L})(p<0.05$, Figure 3(c)).

3.6. Liver Lipid Peroxidation. To assess the tissue lipid peroxidation levels, the MDA concentration in the liver was measured. As shown in Figure 4(a), the concentration of MDA was significantly lower in the SP group (11.69 \pm $0.95 \mathrm{mmol} / \mathrm{mg}$ protein) than in the control group (12.77 \pm $1.11 \mathrm{mmol} / \mathrm{mg}$ protein $)(p<0.05)$.

3.7. Liver Neutrophil Accumulation. The neutrophil accumulation level in the liver was measured by determining the MPO activity. The MPO activity in the liver was significantly reduced in the SP group compared with the control group $(p<0.05$, Figure 4(b)).
3.8. Hepatic Levels of Inflammatory Cytokines. The increase in IL-6 in the liver was significantly suppressed in the SP group compared with the control group ( $p<0.05$, Figure 5(a)). The level of TNF- $\alpha$ in the liver was also lower in the SP group than in the control group $(p<0.05$, Figure 5(b)). These results suggest that SP can reduce the systemic release of inflammatory cytokines compared with the control group during RBC storage.

3.9. Liver Histology. As shown in Figure 6, the livers in the control group exhibited neutrophil infiltration, but these phenomena were less pronounced in the SP livers. The inflammation scores were significantly lower in the SP group than in the control group $(p<0.05$, Figure 6(c)).

\section{Discussion}

In this study, the effects of adding SP during RBC storage on the storage lesions of RBCs in vitro and the organ injury after transfusion were evaluated in a murine model. Our experiments indicated that SP can restore the oxygencarrying capacity of RBCs during storage and decrease the AST activity, BUN concentration, and LDH activity in the plasma of the recipients $2 \mathrm{~h}$ after the transfusion of stored RBCs. The addition of SP during RBC storage also decreased the MDA concentration, the MPO activity, and the IL- 6 and TNF- $\alpha$ levels in the liver after transfusion compared with the control group. Taken together, our findings suggest that the addition of SP to stored RBCs can attenuate RBC storage lesions and subsequent liver injury in a murine transfusion model.

SP may benefit the oxygen-carrying capacity of RBCs because it not only provides a substrate for energy metabolism but also acts as an antioxidant during RBC storage. Pyruvate is the key intermediate product of the glycometabolism pathway and can be catalysed into lactate, promoting the generation of ATP and 2,3-DPG [35]. A previous study indicated that the ATP and 2,3-DPG levels in RBCs significantly decrease during storage [16]. SP may restore the oxygencarrying capacity of stored RBCs by improving energy metabolism. In addition, the oxygen-carrying capacity of RBCs is decreased when haemoglobin is oxidized to methemoglobin [36]. SP may restore the oxygen-carrying capacity of RBCs by attenuating the oxidation of haemoglobin during $\mathrm{RBC}$ storage.

The method of directly labelling RBCs with FITC has been reported previously [30]. We measured the 24-h survival of fresh RBCs (Table 1S (in Supplementary Material available online at http://dx.doi.org/10.1155/2016/3549207), 97.6 $\pm 1.2 \%$ ), and it was similar to the survival reported in a previous study [25]. This result indicated that the FITClabelling protocol did not negatively affect RBC viability.

The protective effect of sodium pyruvate on RBCs stored in CPDA-1 versus more modern storage solutions (i.e., SAGM, AS-1) still needs to be evaluated. In this study, we reported that the addition of sodium pyruvate to RBCs during storage in CPDA-1 has a beneficial effect on storage lesions in vitro and subsequently alleviates liver injury following transfusion of the stored RBCs in vivo. This beneficial effect 


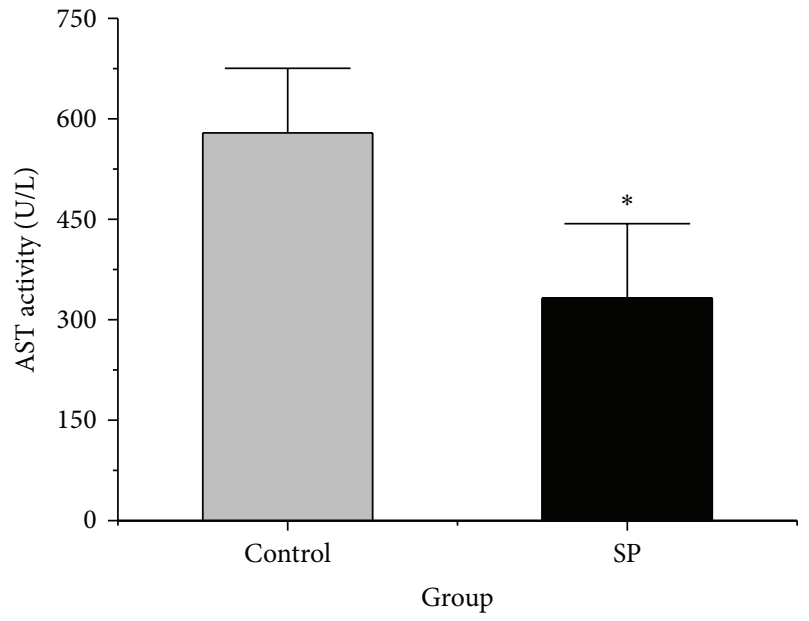

(a)

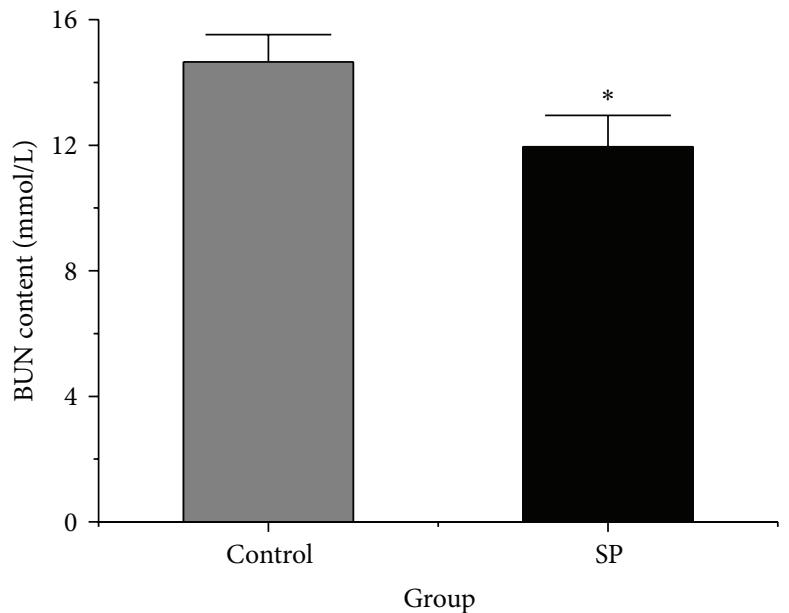

(b)

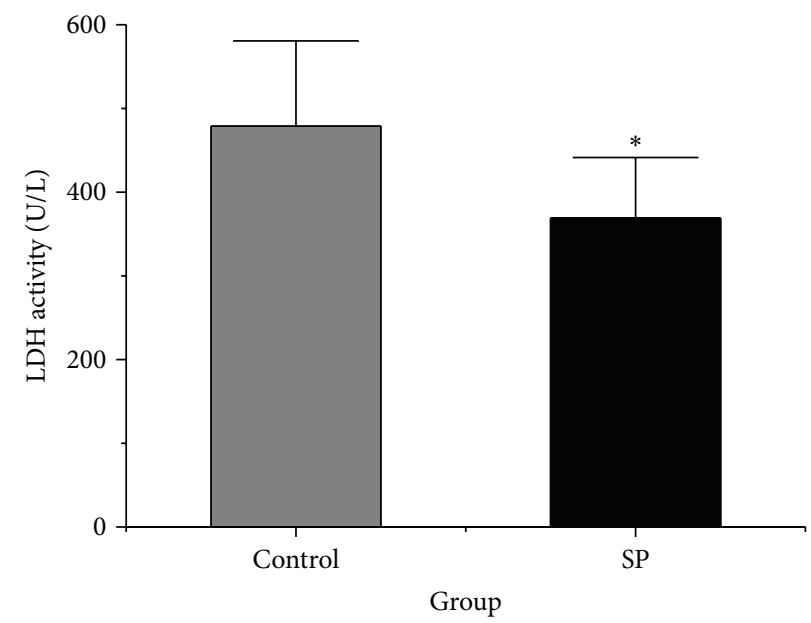

(c)

FIgURe 3: Plasma AST activity, BUN concentration, and LDH activity. (a) Plasma AST activity. (b) Plasma BUN concentration. (c) Plasma $\mathrm{LDH}$ activity. The data are plotted as the means $\pm \mathrm{SD}(n=10) .{ }^{*} p<0.05$ versus the control group.

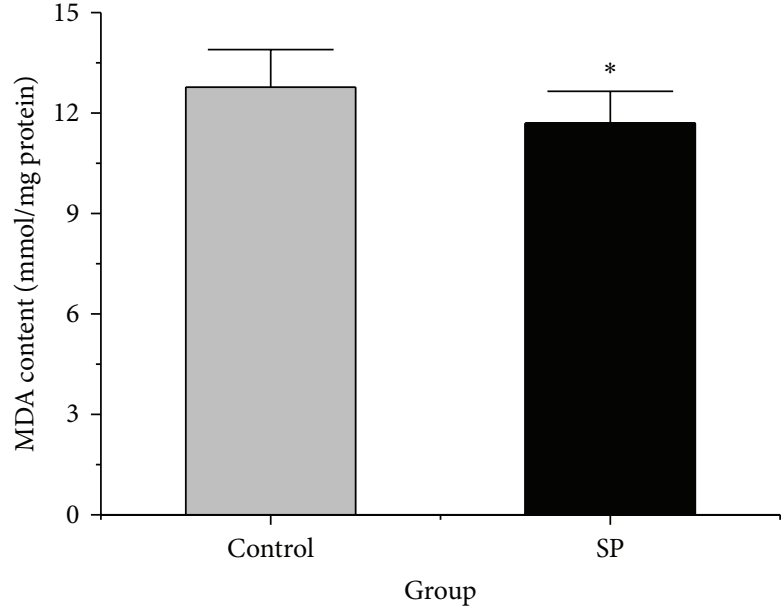

(a)

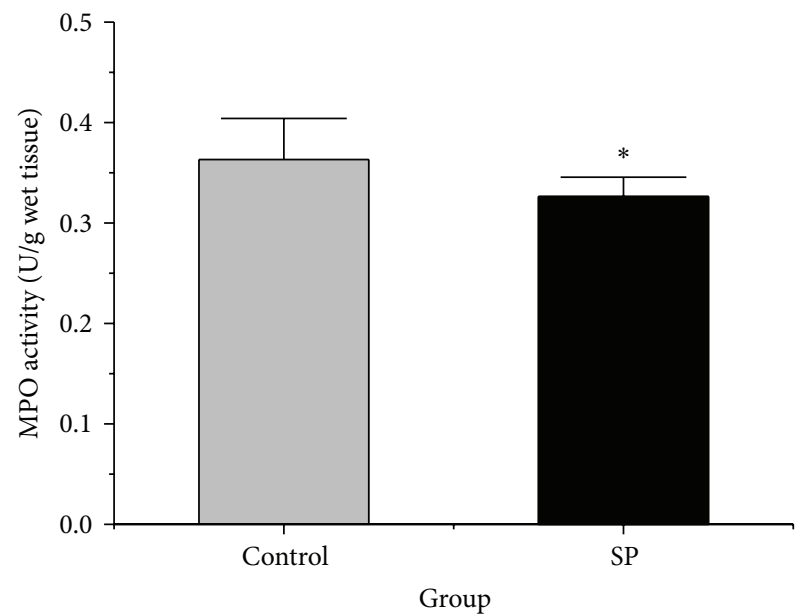

(b)

FIGURE 4: MDA and MPO levels in the livers of the two groups. (a) Liver MDA concentration of the two groups $(n=10)$. (b) Liver MPO activity of the two groups $(n=10)$. The data are plotted as the means \pm SD. ${ }^{*} p<0.05$ versus the control group. 


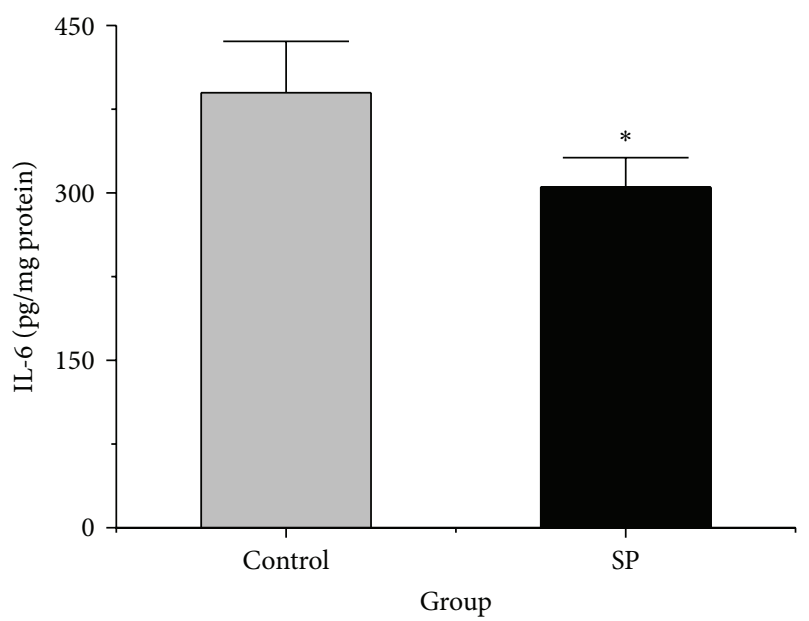

(a)

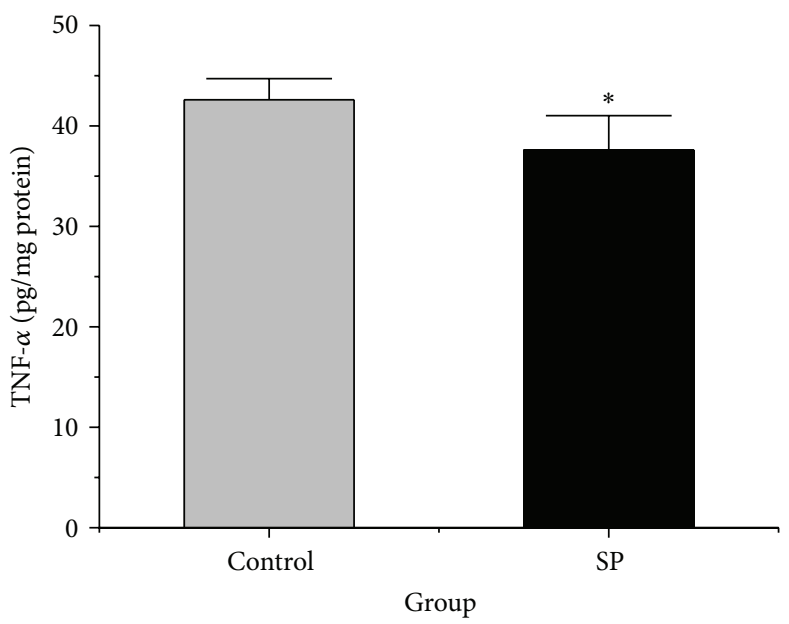

(b)

FIGURE 5: Levels of IL-6 and TNF- $\alpha$ in liver of the two groups. (a) Liver IL-6 levels of the two groups $(n=10)$. (b) Liver TNF- $\alpha$ levels of the two groups $(n=10)$. The data are plotted as the means \pm SD. ${ }^{*} p<0.05$ versus the control group.

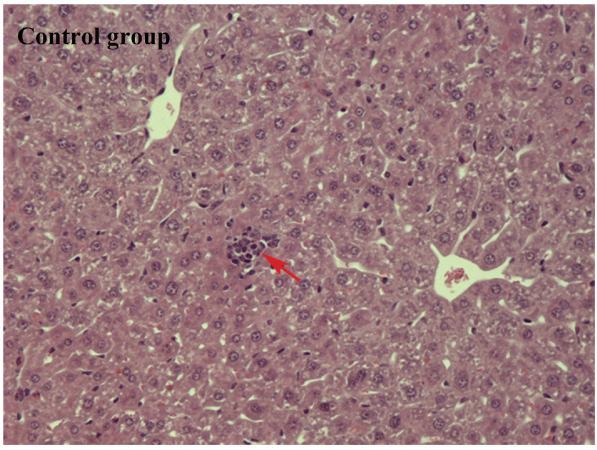

(a)

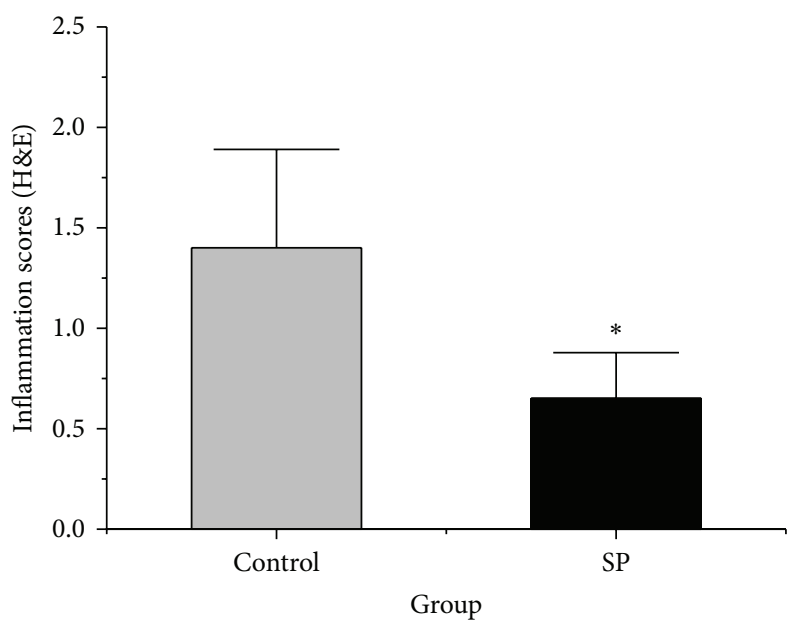

(c)

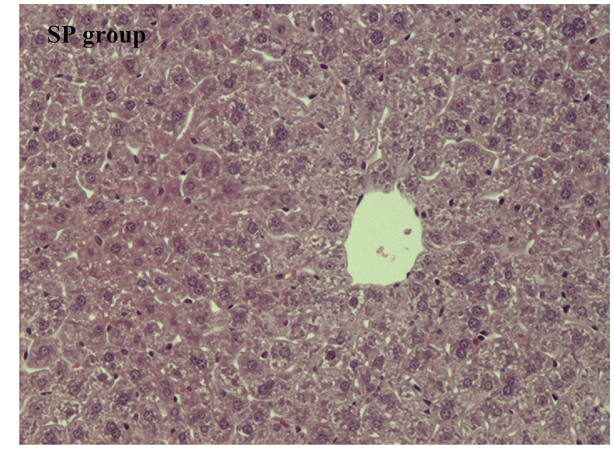

(b) 
may be due to an improvement in energy metabolism, oxidative state, intracellular acidosis, or the oxygen-carrying capacity of the RBCs $[20,37]$. Both SAGM and AS-1 solutions contain mannitol and sodium chloride, which can maintain the integrity of the cell membrane, while the CPDA-1 solution does not. However, SAGM and AS-1 storage solutions do not contain ingredients that specifically target reactive oxygen species, intracellular acidosis, or energy metabolism. Thus, we believe that the addition of sodium pyruvate to the modern storage solutions can still provide additional protective effects to the RBCs.

Addition of sodium pyruvate to stored RBCs has been investigated previously for the purpose of improving the quality of stored human RBCs. A study by Beutler et al. has indicated that pyruvate can maintain ATP levels during human RBCs storage at concentrations ranging from 0.3 to $3 \mathrm{mM}$, whereas 2,3-DPG cannot [23,38]. Dawson et al. reported that higher pyruvate concentrations $(40-320 \mathrm{mM})$ could maintain elevated or normal 2,3-DPG levels, but decreased ATP levels in stored human RBCs [24]. In our study, the sodium pyruvate dose $(2.5 \mathrm{mM})$ was selected based on previous studies and our preliminary experiment. The reported therapeutic in vitro dose for sodium pyruvate in previous studies has mostly been between 1 and $4 \mathrm{mM}$ [23, $39,40]$. In our preliminary experiment, we observed that $2.5 \mathrm{mM}$ dose was superior for $P_{50}$ values in vitro, compared with a dose of $1 \mathrm{mM}, 5 \mathrm{mM}$, or $10 \mathrm{mM}$. We found the pyruvate (2.5 mM) could improve the ATP levels [41] and oxygencarrying capacity of stored murine RBCs. More studies are required to optimize the pyruvate concentration for improving the quality of stored RBCs. In addition, previous studies have indicated that sodium pyruvate can attenuate tissue damage in haemorrhagic shock, brain damage, and ischemia-reperfusion injury models. Therefore, the washing procedure prior to transfusion in this study was necessary to exclude the impact of residual sodium pyruvate on liver injury.

A previous study indicated that prolonged $\mathrm{RBC}$ storage prior to transfusion is associated with increased rates of infection, multiorgan failure, and mortality in some hospitalized patients [11, 42-44]. Although the precise mechanisms responsible for the deleterious effects of stored RBC transfusion are not fully understood, the reduced deformability of stored RBCs has been considered an important reason for the negative effects observed after the transfusion of stored RBCs [45]. In this study, we found that the addition of SP to stored RBCs can attenuate liver injury after transfusion. The improvement of the membrane deformability of RBCs during storage may contribute to the beneficial effects of SP. SP may improve the membrane deformability of RBCs and maintain the integrity of the $\mathrm{RBC}$ membrane by reducing lipid peroxidation. Additional studies should clarify the precise mechanisms through which SP protects organs in the recipient after the transfusion of stored RBCs.

Although only liver injury was evaluated in this study, the addition of SP to stored RBCs may also mitigate nonhepatic organ injury. Our results indicate that the addition of SP to stored RBCs can decrease the plasma BUN concentration (Figure 1S), a conventional marker of kidney injury, after transfusion. In addition, $\mathrm{LDH}$ is an important isoenzyme in glycolysis and ubiquitous in the lung, heart, liver, and other tissues. Tissue injury causes the release of $\mathrm{LDH}$ into the plasma, which increases the LDH activity in plasma [46]. In this study, the addition of SP to stored RBCs can also inhibit the $\mathrm{LDH}$ activity in plasma after transfusion. This result indicates that the addition of SP to stored RBCs may attenuate tissue injury in the recipient after transfusion.

The addition of SP to saline alters the osmolality of the final solution. Previous studies reported that the osmotic pressure can affect the quality of stored RBCs [47, 48]. Increases in osmolality may reportedly promote the leakage of potassium, disturbing the ionic equilibrium, which consequently decreases the quality of stored RBCs [49]. In this experiment, the addition of SP to saline slightly elevated the osmolality of the final solution (SP group: $376 \pm 2 \mathrm{mOsm} / \mathrm{kg}$; control group: $368 \pm 1 \mathrm{mOsm} / \mathrm{kg}$ ), but SP was found to benefit stored RBCs. These results indicate that changes in the osmolality due to the addition of SP during RBC storage may not contribute to the beneficial effects of SP observed in this study.

In this study, the murine system is a tractable platform in which to rapidly study the adverse consequences of transfusion of stored RBCs in vivo and the improvement of adding $\mathrm{SP}$ during RBC storage. However, in view of the difference between mouse model and human in RBC storage, these observations from mouse model may not always be directly applicable to humans [50-52]. Thus subsequent studies are still needed to test these hypotheses in human RBC storage systems.

\section{Conclusions}

In conclusion, the addition of SP solution to RBCs during storage restores the oxygen-carrying capacity of RBCs and subsequently alleviates liver injury after the transfusion of stored RBCs. Although these findings warrant further study, our results indicate that the addition of SP to stored RBCs may be a promising strategy for avoiding the adverse effects of transfusing stored RBCs. Subsequent focused studies in humans will be required to test these hypotheses in human $\mathrm{RBC}$ storage systems.

\section{Abbreviations}

AST: Aspartate aminotransferase

BUN: Blood urea nitrogen

CPDA-1: Citrate-phosphate-dextrose-adenine-1

FITC: Fluorescein isothiocyanate

FDA: Food and Drug Administration

IL-6: Interleukin-6

MDA: Malondialdehyde

MPO: Myeloperoxidase

LDH: Lactate dehydrogenase

$\mathrm{pCO}_{2}$ : Partial pressure of carbon dioxide

$\mathrm{pO}_{2}$ : $\quad$ Partial pressure of oxygen

RBCs: Red blood cells

ROS: Reactive oxygen species

SOD: Superoxide dismutase 
SP: $\quad$ Sodium pyruvate

TCA: Tricarboxylic acid

TNF- $\alpha$ : Tumor necrosis factor-alpha.

\section{Competing Interests}

The authors declare that they have no conflict of interests.

\section{Authors' Contributions}

Sha Xia and Gan Chen contributed equally to this work: they conceived the study, carried out the studies, and wrote the manuscript. Yujing Yin and Bo Wang participated in the revision of the manuscript and helped to measure inflammatory cytokines levels. Zhenwei Sun helped to draft the manuscript and harvest RBCs from animals. Jingxiang Zhao participated in the revision of the manuscript and helped to draft the manuscript and Penglong Li contributed to the study statistical analyses and helped to transfuse the stored RBCs. Lian Zhao and Hong Zhou are the principal investigators and take responsibility for all conceptual and technical aspects of this study. All authors have read and approved the final manuscript.

\section{Acknowledgments}

This work was supported by grants from the National Natural Science Foundation of China (no. 81600148) and the Youth Science Fund of AMMS (no. 2015CXJJ22).

\section{References}

[1] G. J. C. G. M. Bosman, J. M. Werre, F. L. A. Willekens, and V. M. J. Novotný, "Erythrocyte ageing in vivo and in vitro: structural aspects and implications for transfusion," Transfusion Medicine, vol. 18, no. 6, pp. 335-347, 2008.

[2] I. Chin-Yee, N. Arya, and M. S. d'Almeida, “The red cell storage lesion and its implication for transfusion," Transfusion Science, vol. 18, no. 3, pp. 447-458, 1997.

[3] C. F. Hogman and H. T. Meryman, "Storage parameters affecting red blood cell survival and function after transfusion," Transfusion Medicine Reviews, vol. 13, no. 4, pp. 275-296, 1999.

[4] S. R. Stowell, N. H. Smith, J. C. Zimring et al., "Addition of ascorbic acid solution to stored murine red blood cells increases posttransfusion recovery and decreases microparticles and alloimmunization," Transfusion, vol. 53, no. 10, pp. 2248-2257, 2013.

[5] J. A. Cancelas, L. J. Dumont, L. A. Maes et al., "Additive solution7 reduces the red blood cell cold storage lesion," Transfusion, vol. 55, no. 3, pp. 491-498, 2015.

[6] A. D’Alessandro, G. Liumbruno, G. Grazzini, and L. Zolla, "Red blood cell storage: the story so far," Blood Transfusion, vol. 8, no. 2, pp. 82-88, 2010.

[7] E. Bennett-Guerrero, T. H. Veldman, A. Doctor et al., "Evolution of adverse changes in stored RBCs," Proceedings of the National Academy of Sciences of the United States of America, vol. 104, no. 43, pp. 17063-17068, 2007.

[8] I. Corteś-Puch, D. Wang, J. Sun et al., "Washing older blood units before transfusion reduces plasma iron and improves outcomes in experimental canine pneumonia," Blood, vol. 123, no. 9, pp. 1403-1411, 2014.

[9] E. A. Hod, N. Zhang, S. A. Sokol et al., "Transfusion of red blood cells after prolonged storage produces harmful effects that are mediated by iron and inflammation," Blood, vol. 115, no. 21, pp. 4284-4292, 2010.

[10] P. C. Spinella, C. L. Carroll, I. Staff et al., "Duration of red blood cell storage is associated with increased incidence of deep vein thrombosis and in hospital mortality in patients with traumatic injuries," Critical Care, vol. 13, no. 5, article R151, 2009.

[11] G. Zallen, P. J. Offner, E. E. Moore et al., "Age of transfused blood is an independent risk factor for postinjury multiple organ failure," American Journal of Surgery, vol. 178, no. 6, pp. 570$572,1999$.

[12] L. E. Lögdberg, T. Vikulina, J. C. Zimring, and C. D. Hillyer, "Animal models of transfusion-related acute lung injury," Transfusion Medicine Reviews, vol. 23, no. 1, pp. 13-24, 2009.

[13] L. J. Dumont and J. P. AuBuchon, "Evaluation of proposed FDA criteria for the evaluation of radiolabeled red cell recovery trials," Transfusion, vol. 48, no. 6, pp. 1053-1060, 2008.

[14] J. R. Hess, "An update on solutions for red cell storage," Vox Sanguinis, vol. 91, no. 1, pp. 13-19, 2006.

[15] R. S. Sousa, R. A. Barrêto-Júnior, I. K. F. Sousa et al., "Evaluation of hematologic, blood gas, And select biochemical variables in ovine whole blood stored in CPDA-1 bags," Veterinary Clinical Pathology, vol. 42, no. 1, pp. 27-30, 2013.

[16] J. R. Hess, "Red cell changes during storage," Transfusion and Apheresis Science, vol. 43, no. 1, pp. 51-59, 2010.

[17] F. A. Oski, S. F. Travis, L. D. Miller, M. Delivoria-Papadopoulos, and E. Cannon, "The in vitro restoration of red cell 2,3diphosphoglycerate levels in banked blood," Blood, vol. 37, no. 1, pp. 52-58, 1971.

[18] S. D. Varma, K. R. Hegde, and S. Kovtun, "Attenuation and delay of diabetic cataracts by antioxidants: effectiveness of pyruvate after onset of cataract," Ophthalmologica, vol. 219, no. 5, pp. 309315, 2005.

[19] P. N. Slovin, C.-J. Huang, J. R. Cade et al., "Sodium pyruvate is better than sodium chloride as a resuscitation solution in a rodent model of profound hemorrhagic shock," Resuscitation, vol. 50, no. 1, pp. 109-115, 2001.

[20] J. Yang, J. X. Zhao, Y. Wang et al., "Effects of sodium pyruvate on ameliorating metabolic acidosis," Artificial Cells, Nanomedicine, and Biotechnology, vol. 44, no. 1, pp. 48-55, 2016.

[21] L.-D. Guan, Z.-L. Wang, L. Zhao et al., "Protective effect of sodium pyruvate on ischemia/reperfusion injury of rats subjected to hemorrhagic shock," Zhongguo Ying Yong Sheng Li Xue Za Zhi, vol. 23, no. 3, pp. 264-268, 2007.

[22] R. Pan, Z. Rong, Y. She, Y. Cao, L.-W. Chang, and W.-H. Lee, "Sodium pyruvate reduces hypoxic-ischemic injury to neonatal rat brain," Pediatric Research, vol. 72, no. 5, pp. 479-489, 2012.

[23] N. V. Paniker and E. Beutler, "Pyruvate effect in maintenance of ATP and 2,3-DPG of stored blood," The Journal of Laboratory and Clinical Medicine, vol. 78, no. 3, pp. 472-482, 1971.

[24] R. B. Dawson, R. T. Hershey, and C. S. Myers, "Blood preservation XXIX. Pyruvate maintains normal red cell 2,3-DPG for six weeks of storage in CPD-adenine," Transfusion, vol. 20, no. 2, pp. 218-223, 1980.

[25] B. Yu, C. Lei, D. M. Baron, A. U. Steinbicker, K. D. Bloch, and W. M. Zapol, "Diabetes augments and inhaled nitric oxide prevents the adverse hemodynamic effects of transfusing syngeneic stored blood in mice," Transfusion, vol. 52, no. 7, pp. 1410-1422, 2012. 
[26] C. R. Gilson, T. S. Kraus, E. A. Hod et al., "A novel mouse model of red blood cell storage and posttransfusion in vivo survival," Transfusion, vol. 49, no. 8, pp. 1546-1553, 2009.

[27] A. T. Makley, M. D. Goodman, L. A. W. Friend et al., "Murine blood banking: characterization and comparisons to human blood," Shock, vol. 34, no. 1, pp. 40-45, 2010.

[28] M. S. d'Almeida, J. Jagger, M. Duggan, M. White, C. Ellis, and I. H. Chin-Yee, "A comparison of biochemical and functional alterations of rat and human erythrocytes stored in CPDA1 for 29 days: implications for animal models of transfusion," Transfusion Medicine, vol. 10, no. 4, pp. 291-303, 2000.

[29] V. Han, K. Serrano, and D. V. Devine, "A comparative study of common techniques used to measure haemolysis in stored red cell concentrates," Vox Sanguinis, vol. 98, no. 2, pp. 116-123, 2010.

[30] H. H. Lipowsky, L. E. Cram, W. Justice, and M. J. Eppihimer, "Effect of erythrocyte deformability on in vivo red cell transit time and hematocrit and their correlation with in vitro filterability," Microvascular Research, vol. 46, no. 1, pp. 43-64, 1993.

[31] K. Parthasarathi and H. H. Lipowsky, "Capillary recruitment in response to tissue hypoxia and its dependence on red blood cell deformability," American Journal of Physiology-Heart and Circulatory Physiology, vol. 277, no. 6, pp. H2145-H2157, 1999.

[32] G. Chen, G. You, Y. Wang et al., "Effects of synthetic colloids on oxidative stress and inflammatory response in hemorrhagic shock: comparison of hydroxyethyl starch 130/0.4, hydroxyethyl starch 200/0.5, and succinylated gelatin," Critical Care, vol. 17, article R141, 2013.

[33] X. Luo, Y. Yin, G. You et al., "Gradually increased oxygen administration improved oxygenation and mitigated oxidative stress after resuscitation from severe hemorrhagic shock," Anesthesiology, vol. 123, no. 5, pp. 1122-1132, 2015.

[34] J. Wu, H. Zhang, H. Zheng, and Y. Jiang, "Hepatic inflammation scores correlate with common carotid intima-media thickness in rats with NAFLD induced by a high-fat diet," BMC Veterinary Research, vol. 10, article 162, 2014.

[35] E. K. Meyer, D. F. Dumont, S. Baker, and L. J. Dumont, "Rejuvenation capacity of red blood cells in additive solutions over long-term storage," Transfusion, vol. 51, no. 7, pp. 1574-1579, 2011.

[36] P. Cabrales, M. Intaglietta, and A. G. Tsai, “Transfusion restores blood viscosity and reinstates microvascular conditions from hemorrhagic shock independent of oxygen carrying capacity," Resuscitation, vol. 75, no. 1, pp. 124-134, 2007.

[37] U. N. Das, "Pyruvate is an endogenous anti-inflammatory and anti-oxidant molecule," Medical Science Monitor, vol. 12, no. 5, pp. RA79-RA84, 2006.

[38] E. Beutler and L. A. Wood, "Preservation of red cell 2,3diphosphoglycerate in modified ACD solution and in experimental artificial storage media," Vox Sanguinis, vol. 20, no. 5, pp. 403-413, 1971.

[39] A. K. Salahudeen, E. C. Clark, and K. A. Nath, "Hydrogen peroxide-induced renal injury. A protective role for pyruvate in vitro and in vivo," The Journal of Clinical Investigation, vol. 88, no. 6, pp. 1886-1893, 1991.

[40] J. C. Jagtap, A. Chandele, B. A. Chopde, and P. Shastry, "Sodium pyruvate protects against $\mathrm{H}_{2} \mathrm{O}_{2}$ mediated apoptosis in human neuroblastoma cell line-SK-N-MC," Journal of Chemical Neuroanatomy, vol. 26, no. 2, pp. 109-118, 2003.

[41] S. Xia, G. Chen, Y. Wang et al., "Preliminary study on the improvement of the lesion of stored red blood cells by sodium pyruvate," Chinese Journal of Blood Transfusion, vol. 29, pp. 353356, 2016.
[42] G. J. Murphy, B. C. Reeves, C. A. Rogers, S. I. A. Rizvi, L. Culliford, and G. D. Angelini, "Increased mortality, postoperative morbidity, and cost after red blood cell transfusion in patients having cardiac surgery," Circulation, vol. 116, no. 22, pp. $2544-$ 2552, 2007.

[43] S. Basran, R. Frumento, A. Cohen et al., "The association between duration of storage of transfused red blood cells and morbidity and mortality after reoperative cardiac surgery," Anesthesia and Analgesia, vol. 103, no. 1, pp. 15-20, 2009, Anesthesia and Analgesia, vol. 108, pp. 1953, 2009.

[44] E. C. Vamvakas and M. A. Blajchman, "Transfusion-related mortality: the ongoing risks of allogeneic blood transfusion and the available strategies for their prevention," Blood, vol. 113, no. 15, pp. 3406-3417, 2009.

[45] J.-X. Zhao, B. Wang, G.-X. You et al., "Hypertonic saline dextran ameliorates organ damage in beagle hemorrhagic shock," PLoS ONE, vol. 10, no. 8, Article ID e0136012, 2015.

[46] M. Abe, Y. Takiguchi, S. Ichimaru, K. Tsuchiya, and K. Wada, "Comparison of the protective effect of $\mathrm{N}$-acetylcysteine by different treatments on rat myocardial ischemia-reperfusion injury," Journal of Pharmacological Sciences, vol. 106, no. 4, pp. 571-577, 2008.

[47] G. J. C. G. M. Bosman, J. C. A. Cluitmans, Y. A. M. Groenen, J. M. Werre, F. L. A. Willekens, and V. M. J. Novotný, "Susceptibility to hyperosmotic stress-induced phosphatidylserine exposure increases during red blood cell storage," Transfusion, vol. 51, no. 5, pp. 1072-1078, 2011.

[48] H. G. Jarvis, D. M. Gore, C. Briggs, M. C. Chetty, and G. W. Stewart, "Cold storage of 'cryohydrocytosis' red cells: the osmotic susceptibility of the cold-stored erythrocyte," British Journal of Haematology, vol. 122, no. 5, pp. 859-868, 2003.

[49] J. Hirayama, H. Abe, H. Azuma, and H. Ikeda, "Leakage of potassium from red blood cells following gamma ray irradiation in the presence of dipyridamole, trolox, human plasma or mannitol," Biological and Pharmaceutical Bulletin, vol. 28, no. 7, pp. 1318-1320, 2005.

[50] K. Prestia, S. Bandyopadhyay, A. Slate et al., "Transfusion of stored blood impairs host defenses against Gram-negative pathogens in mice," Transfusion, vol. 54, no. 11, pp. 2842-2851, 2014.

[51] J. C. Zimring and S. L. Spitalnik, "On the appropriate use and interpretation of animal models in transfusion medicine research," Transfusion, vol. 53, no. 10, pp. 2334-2339, 2013.

[52] E. A. Hod, "Red blood cell transfusion-induced inflammation: myth or reality," ISBT Science Series, vol. 10, pp. 188-191, 2015. 


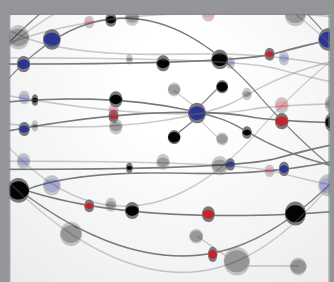

The Scientific World Journal
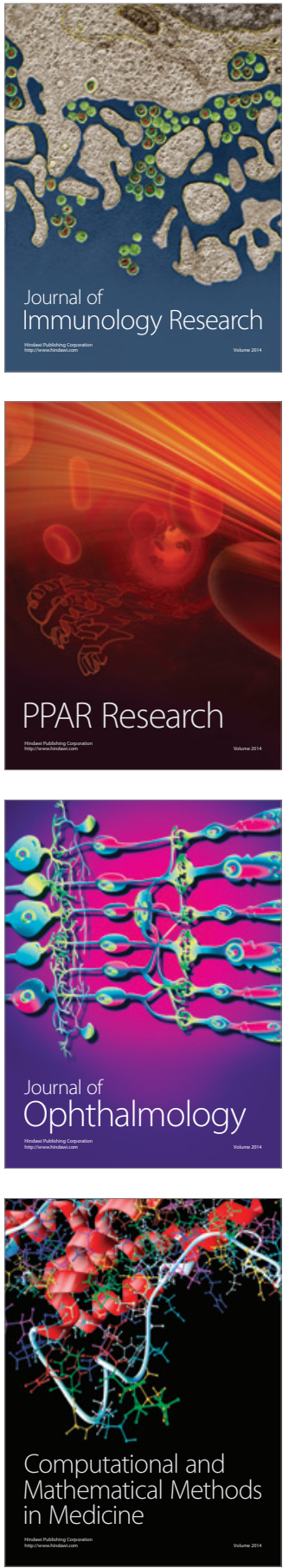

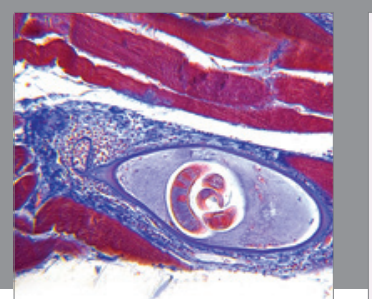

Gastroenterology Research and Practice

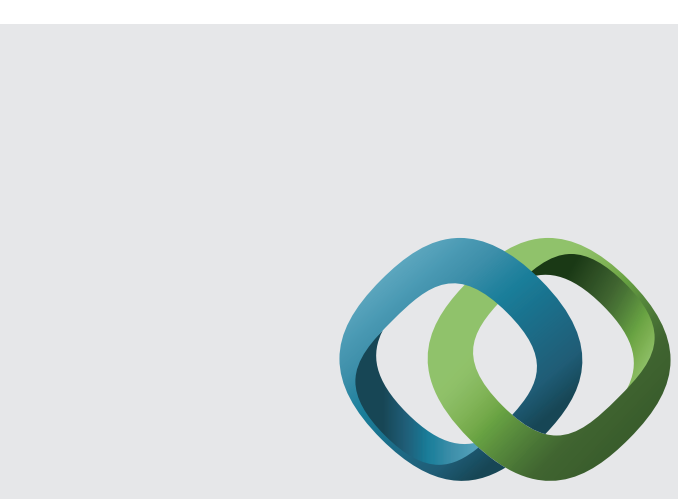

\section{Hindawi}

Submit your manuscripts at

http://www.hindawi.com
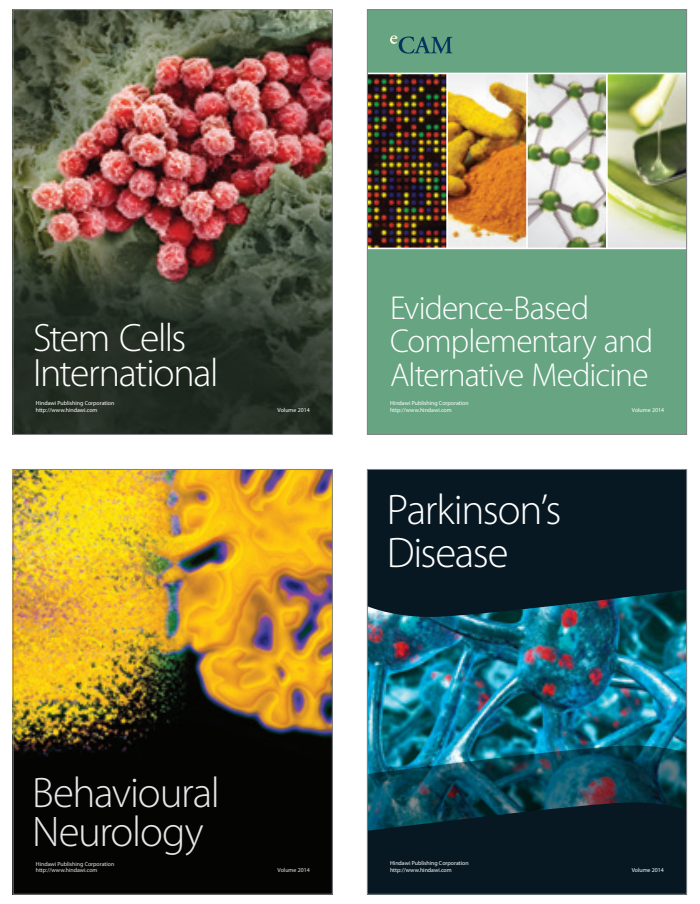
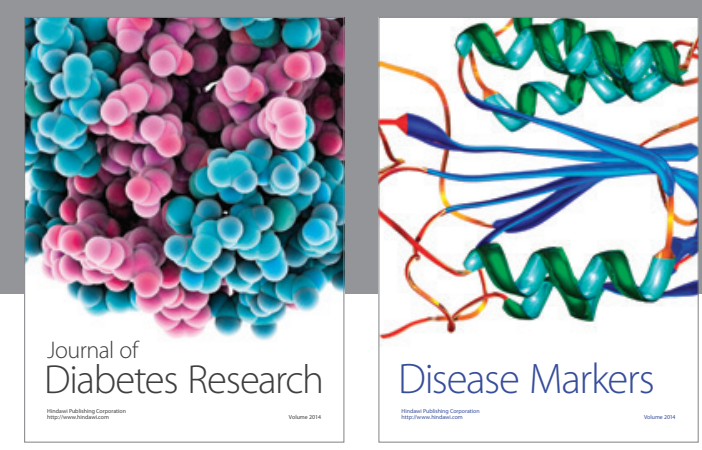

Disease Markers
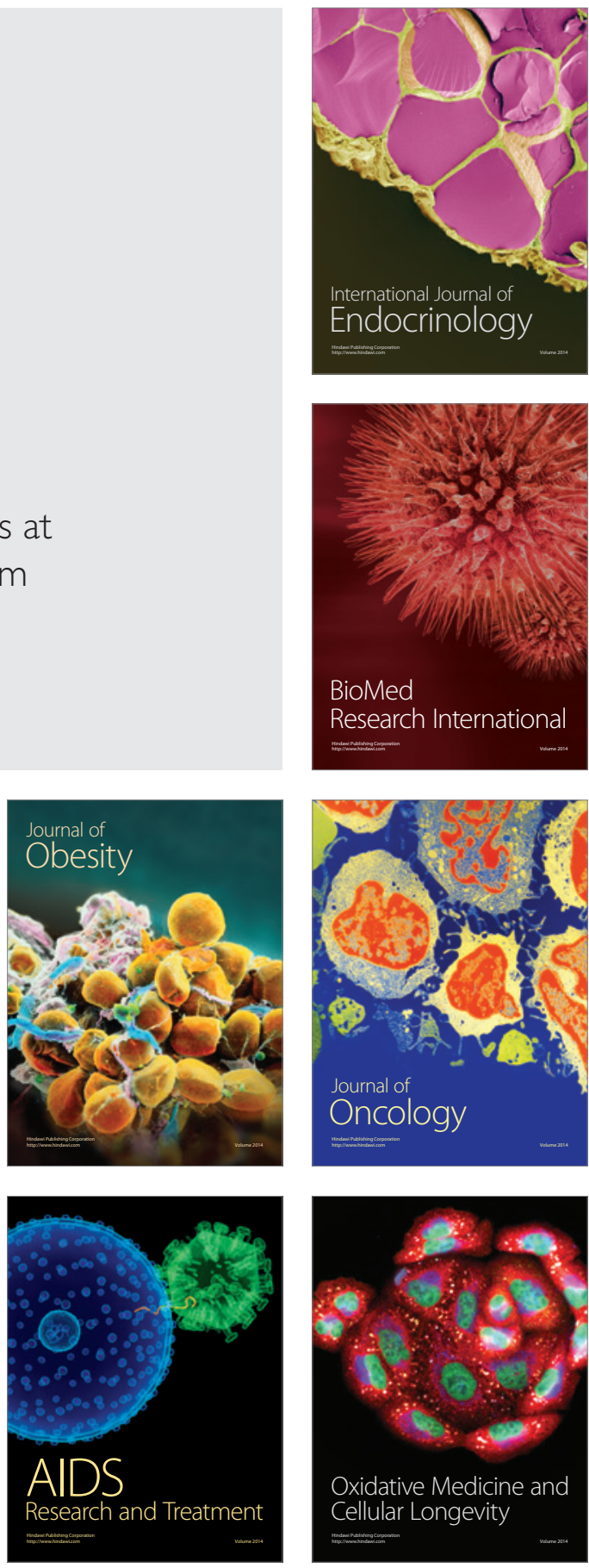